\title{
Carl Einstein und der Mythos
}

Carl Einstein et le mythe

Carl Einstein and the Myth

\section{Klaus H. Kiefer}

\section{OpenEdition}

\section{Journals}

Édition électronique

URL : http://journals.openedition.org/ceg/4432

DOI : $10.4000 /$ ceg. 4432

ISSN : 2605-8359

\section{Éditeur}

Presses Universitaires de Provence

Édition imprimée

Date de publication : 2 mai 2019

Pagination : 95-108

ISBN : 979-10-320-0214-8

ISSN : 0751-4239

\section{Référence électronique}

Klaus H. Kiefer, "Carl Einstein und der Mythos“, Cahiers d'Études Germaniques [Online], 76 | 2019, Online erschienen am: 02 November 2020, abgerufen am 25 Januar 2021. URL: http:// journals.openedition.org/ceg/4432 ; DOI: https://doi.org/10.4000/ceg.4432 


\section{Carl Einstein und der Mythos}

Klaus H. KIEFER

Ludwig-Maximilians-Universität München

Wie lange hab' ich nicht am Wahn hinausgekehrt Und nie wird's rein, das ist doch unerhört! (Goethe, Faust I, V. 4162f.)

\section{Mythos und Information}

Im Wesentlichen beruht Mythos auf Informationsdefiziten... Diese pointiert modernistische Ansicht ist insofern richtig, als Mythos und Logos seit der Antike (Plato) um ihre Vorherrschaft in der jeweiligen Weltanschauung ringen und ihre claims abstecken, und ohne Zweifel ist unser Erkenntnisziel heute der Information, dem Faktum verpflichtet. Wir wissen freilich auch, wie trügerisch über die Zeiten die Vernunft ${ }^{1}$ sein konnte (und sie ist es noch), während im Mythos der Irrtum keine bedeutsame Rolle spielt. Mythen halten sich als Moralspender von alters her und nicht zuletzt auch ihrer Ästhetizität wegen. Indes ist die Eingangsthese durchaus problematisch: schon mit dem Austritt der menschlichen Spezies aus dem Reiz/Reaktions-Muster der tierischen Kommunikation hat jedes Zeichen eine Bewährungsprobe zu bestehen. Im Prinzip ist es - situationsabstrakt, arbiträr, wie es ist - mythisch, so dass über weite Strecken Philosophie Sprachkritik zu leisten hat.

Dass der Mythos eine große und (via ,sakraler' Leitmotive) zusammenhängende Erzählung sei, wie es die Postmoderne wissen will, verpasst diesen Vorüberlegungen eine kollektive Dimension, denn just mit der Kodifizierung des Zeichens geht seine Konventionalisierung einher, und dieser unabdingbare Glauben spielt im Großen wie im Kleinen der Kommunikation eine Rolle. Der religiöse oder der politische Mythos, der Mythos (im Kollektivsingular), ist totalitär; unproblematisch dagegen alles andere, was man ,mythisch“ nennen mag, denn jedes Wesen, jedes Ding hat

1. Ich kann hier nur sehr pauschal auf meine Studien zu Aufklärung und Okkultismus verweisen, vgl. Kiefer, „Die famose Hexen-Epoche“ - Sichtbares und Unsichtbares in der Aufklärung. Kant - Schiller - Goethe - Swedenborg - Mesmer - Cagliostro, München, Oldenbourg (Ancien Régime, Aufklärung, Revolution, Bd. 36), 2004. 
sein Wunderbares. Im Mythos - und Religion ist nichts anderes ${ }^{2}$ - erhält Glauben eine Übermacht; die Philologie muss dagegen der semiotischen Problematik, die sie nicht hintergehen kann, ständig Rechnung tragen. Zwischen den „obersten Werten" (Nietzsche), die sich wie auch immer herauskristallisiert haben, und den Zeichenfunktionen (Arbitrarität/Konvention) wogt ein ,Kampf“: „Tout effort artistique vise que l'objet ne soit plus la prémisse fatale, mais le résultat créé par nos actes imaginatifs." (W 4, 182)

\section{Annäherungen an den Mythos}

\section{Gottes Tod, Satire und Politik}

Aus der Distanz eines halben Jahrhunderts - in dem sich die oben genannten Konflikte mitnichten beruhigt haben ${ }^{3}$ - lassen sich im Zeitraum der einsteinschen Vita einige Strukturen erkennen. Nachdem Friedrich Nietzsche den jüdischchristlichen Gott für tot erklärt hatte, sucht die abendländische Intelligenzija, und nicht nur diese, nach Ersatz. Das Wunderbare ist dem jungen Einstein durchaus wertvoll (vgl. noch Br. 127), gerade auch weil er in seinem Romanerstling, zwischen 1906 und 1912 entstanden, Bebuquin oder Die Dilettanten des Wunders, moderne Formen des (religiösen) Mirakels: Lunapark, Alkohol, Zirkus etc., ad absurdum führt. Seit 1913 publiziert er Teile einer Religionssatire, G.F.R.G. (Gesellschaft für religiöse Gründungen), die er 1918 seiner Sammlung Der unentwegte Platoniker einverleibt. Zum selben Zeitpunkt stellt er aber ernsthaft die Frage: „Wo wäre unsere Zentralidee, wo unser Gesetz (anerkennenswert), wo unser Mythus?“ (BA 1, 371), und wiederholt sie nachdrücklich im Europa Almanach 1925 (EA, 116). ${ }^{4}$ Der tote

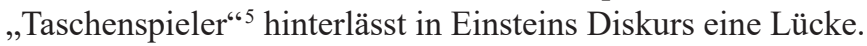

Unbestritten, dass die Religionen auch nach „Gottes Tod“ fortbestehen ${ }^{6}$ und im großen Kulturkampf 1914-1918 an jeder Front die Waffen segnen. Einen scheinbaren Gemeinschaftsersatz bot ja der „Heilige Krieg“ (la Grande Guerre), der für viele in der Kameradschaft der Schützengräben endet. Angeblich war auch ein Fronterlebnis Auslöser für Einsteins Drama Die schlimme Botschaft, ein nietzscheanisches „Dysangelium“ (KSA 6, 211), für das er 1922 wegen Gotteslästerung verurteilt

2. Die Unterscheidung von Religion und Mythos und der Versuch, diesen an primitiven und antiken Kulturen festzumachen, stellt eine diskursive Verzerrung dar, verursacht durch kirchliche Orthodoxie.

3. Vor allem der Islam wirft die okzidentale Religionskritik (die Kritik an den judäo-christlichen Mythen) um zwei Jahrhunderte zurück, indem es die Errungenschaften der Aufklärung, nämlich die Gedanken- und Glaubensfreiheit, wieder einer dualen Kategorisierung (gläubig vs. ungläubig) unterwirft. Diese „Soumission“ (Houellebecq) ist intolerabel. Auch angesichts fremdkultureller Sitten und Gebräuche darf der Verstand nicht stillstehen.

4. Möglich, dass mit „Gesetz“ die jüdische Tora zu assoziieren ist, was jedoch keine weitere Bedeutung hätte. Gott und Tora bilden bei Einstein gleicherweise eine diskursive Leerstelle, in seinen Worten: eine Wunde mit einem „zuckenden Klebpflaster“ (BA 1, 105).

5. Einstein zitiert offenbar Apollinaires „enchanteur pourrissant“ (CEA, 17).

6. Der von Obsessionen des Wahnsinns und des Todes geplagte junge Einstein experimentiert wohl unter dem Einfluss von Franz Blei mit ,neokatholischen“ Ideen; das reicht von „Der Papst ist der schlechthin Gottvergessene“ bis zu „Papst ist Gott“(W 4, 130). 
wurde. Hier werden Aspekte der Mythenbildung im wahrsten Sinn des Wortes , durchgespielt‘.

Im Kontext der „Urkatastrophe“ des 20. Jahrhunderts machen sich zwei neue Kräfte in unterschiedlicher Weise bemerkbar: Seit Karl Marx forderte, die Welt nicht mehr nur zu interpretieren, sondern handelnd zu verändern (was im Grunde auch den christlichen Geboten, ja dem Konventionsdruck jeden Zeichens inhärent ist), entsteht ein wirkungsmächtiger Mythos, dem man die mindernde Bezeichnung ,Ideologie nur beimessen sollte, wenn man bereit ist, alle „Überbauten“ als Ideologien zu bezeichnen. Vermutlich erst spät stößt Einstein auf die Theorie Georges Sorels, der die performative Kraft sozialer Mythen („Mittel zur Tat“ [FF, 43]) herausgearbeitet hatte. Dabei kommt es nicht auf den Inhalt, sondern lediglich auf die Stärke der Illusion an. Der Bruch mit der Sowjetideologie kam jedenfalls bald, früher als bei den Surrealisten. Am 23. März 1923 schreibt Einstein an Tony Simon-Wolfskehl:

In Russland ist die Situation insofern schwierig - als jetzt eine verschärfte Censur herrscht. Da man ökonomisch stark nachgibt, eröffnet man die ideologische Offensive - eine politische Censur, die bis zu Lenin geht; der ich mich aber nie fügen würde. Kommt hinzu, dass ich wohl einige bissige Bemerkungen gegen Lunartscharsky, den Minister für Volksaufklärung, und einige seiner Bonzen gemacht habe. Ça m'est égal. Ich lasse mich nicht terrorisieren, selbst nicht von Leuten, mit denen ich sehr sympatisiere. (Br. 170)

\section{Primitive}

Etwas verhaltener setzt die Rezeption der sog. primitiven Kulturen ein, relevant zunächst nicht in der Ethnologie (ehedem „Völkerkunde“), sondern in der Ästhetik. Carl Einsteins Negerplastik markiert 1915 den Wendepunkt. Die afrikanische und auch ozeanische Kunst wirken revolutionär auf die moderne Kunst, so dass sich hier ein Glaube an die mythischen Ingredienzien der Primitive bilden konnte. Hoffte Einstein, dass sich die Sammlung von afrikanischen Kunst- und Kultobjekten, Liedern und „Legenden“ (wie er sie nennt) zu einem elementaren Mythos fügen ließe, der ein Modell für die Moderne abgäbe und damit die mythische Altlast Europas abzulösen vermochte? Er bemerkt schon bald, dass sowohl ausreichende Zeugnisse fehlen, als auch das vorhandene mythische Material zu heterogen ist (BA 2, 63f.); dies auch, als er in seiner Zeitschrift Documents den Focus auf sämtliche archaische Kulturen der Welt öffnet. ${ }^{7}$ In Gesprächen mit Gottlieb Friedrich Reber taucht möglicherweise die Idee eines universalen Mythos an seinem geistigen Horizont auf, ${ }^{8}$ während die letzten theoretischen Entwürfe, zu denen er ansetzt, eher geschichtlicher Natur waren, und nicht eben mythischer. ${ }^{9}$ Einstein verfolgt die

7. Vgl. Kiefer, „Carl Einsteins ,Surrealismus“ - ,Wort von verkrachtem Idealismus übersonnt““, in Isabel Fischer u. Karina Schuller (Hrsg.), Der Surrealismus in Deutschland (?). Interdisziplinäre Studien, Münster, MV Wissenschaft (Wissenschaftliche Schriften der Westfälischen WilhelmsUniversität, Reihe 12: Philologie, Bd. 17), 2016, S. 49-83 sowie ders., „Carl Einstein et le surréalisme - entre les fronts et au-dessus de la mêlée (Bataille, Breton, Joyce)“, [http://melusine-surrealisme.fr/ $\underline{w p / ? p=2069]}$. Stand 30. November 2017.

8. „Reber betrachtet die Moderne sub specie aeternitatis [...]“ (BA 3, 122).

9. Vgl. auch Kap. 5: Einsteins Arbeit am personalen Mythos „Bebuquin“. 
rasch entstehende „Negermode“ mit Missbilligung, obwohl gerade hier, von der kulturellen Peripherie her, eine Weltrevolution begann.

Halten wir nur zwei Momente dieser Dynamik fest: Einstein möchte 1919 wie später auch die Surrealisten um André Breton Kommunismus und Primitive verschmelzen. Seine Begrifflichkeit gleitet vom (expressionistisch glorifizierten) „Armen“ zum proletarischen „Primitiven“. In seinem Manifest „Zur primitiven Kunst“ fordert er im Namen der sozialen Revolution die „uns nötige Kollektivkunst“ (BA 2, 27) und trotz einer gegenläufigen Verwissenschaftlichung der primitiven Kunst verpflichtet Einstein die künstlerische Avantgarde vom Kubismus bis zum Surrealismus auf eine Veränderung der gesellschaftlichen Verhältnisse hin. ${ }^{10}$ Zwangsläufig ist diese ,idealistische“ Alternative zur revolutionären Aktion von Resignation konnotiert, nachdem die deutschen Revolutionen in Berlin und München zerschlagen wurden. Im sog. „Kahnweilerbrief“ wird nur noch die „Umnüancierung der Empfindung“ (Br. 127) beansprucht (im Sinne Ernst Machs); mehr und mehr fordert Einstein jedoch die „Erzeugung des Mythos“ als vorrangige Aufgabe der Kunst. ${ }^{11}$ Er räumt sogar der durch und durch ,privaten Mythologie“ $(\mathrm{K} 1,142)$ Paul Klees große Bedeutung ein.

Dieses „romantische“ Projekt zerbricht 1933 jäh an einem konkurrenten Mythos, der besser organisiert war, vor allem schlagkräftiger (und dem Einsteins Freund, Gottfried Benn, kurzfristig zum Opfer fiel). ${ }^{12}$ In der Fabrikation der Fiktionen - die Alliteration verhinderte wohl den Gebrauch des Wortes ,Mythos ${ }^{6}{ }^{13}$ - leistet Einstein eine suizidäre Kritik an der modernen Kunst, zu deren Durchsetzung er ja wesentlich selbst mit beigetragen hatte.

Warum sich Einstein den mythischen Angeboten der zeitgenössischen Religionen, dem Judentum und dem Christentum, verweigerte, ist schwer zu sagen. Wir erfahren davon erst nach 1930, sicher in einer gewissen Stilisierung. Das Kind weigert sich jedenfalls zu beten, so halten es autobiographische Notizen fest (CEA, 7); zugleich ist es „mythoman“ - es lügt. ${ }^{14}$ Den Mythen des klassischen Altertums zieht der Schüler der alten Sprachen die Indianer-Romane Karl Mays vor (BA 3, 155). Das „Suchen in der Negerplastik“, wie es in Franz Werfels Spiegelmensch ${ }^{15}$ heißt, wird zwar nach und nach einer Ethnologisierung zugeführt (dies auch eine geistige

10. So bis zuletzt: Anonym, „Fünf Jahre SDS in Paris. Eine Chronik“, Der deutsche Schriftsteller. Zeitschrift des Schutzverbandes Deutscher Schriftsteller, Nr. 11 (1938): Sonderheft zum Jubiläum des SDS, S. 30-32, hier S. 30: „Diskussionsabend: Prof. Carl Einstein über Kunst als kollektiver Gebrauchsgegenstand“, 14. Oktober 1935.

11. Das erstmalige (noch ironische) Postulat, 1911 in dem Beitrag „Die Verkündigung“, fällt im Rahmen einer Parodie Stefan Georges und seines Kreises (BA 1, 74, hier „Erzeugung des Mythus“).

12. Vgl. Kiefer, „Primitivismus und Avantgarde - Carl Einstein und Gottfried Benn“, Colloquium Helveticum, Bd. 44 (2015): Primitivismus intermedial, S. 131-168.

13. Der Ausdruck könnte von Sigmund Freud inspiriert sein. Einstein hat den Almanach für das Jahr 1927 (Adolf Josef Storfer [Hrsg.], Wien, Psychoanalytischer Verlag) gekannt (CEA, 422) und darin möglicherweise Freuds - kritisch gemeinten - Ausdruck „Fabrikation von Weltanschauungen" registriert.

14. „Das Kind, der Primitive, der Irre, der Schlafende dichten [...]“(W 4, 365, vgl. K 3, 210).

15. Im Original steht das „Suchen“ im Partizip Präsenz, s. Franz Werfel, Spiegelmensch. Magische Trilogie, München, Kurt Wolff, 1920, S. 130. 
Entkolonialisierung), doch die mythischen Kräfte der Primitive waren ,aus der Flasche“. Es geht Einstein um eine „elementare“ Kunst, die jenseits der klassischen Mimesis angesiedelt ist, um einen Affront gegen die verbrauchte und verhasste, historisch obsolete Hochkultur - eine querelle des Anciens et des Modernes, deren die abendländische Zivilisation freilich schon mehrere gesehen hatte. Der (primitive) Mythos besitzt freilich zügellose Kräfte... Francisco de Goya hat dem schon 1799 in einem seiner Caprichos ${ }^{16}$ Ausdruck verliehen (Abb. 1):

Abb. 1: Francisco de Goya: Capricho, Nr. 43: El sueño de la razón produce monstruos, Aquatinta-Radierung 21,3 x 15,1 cm, 1799, Museo del Prado, Madrid

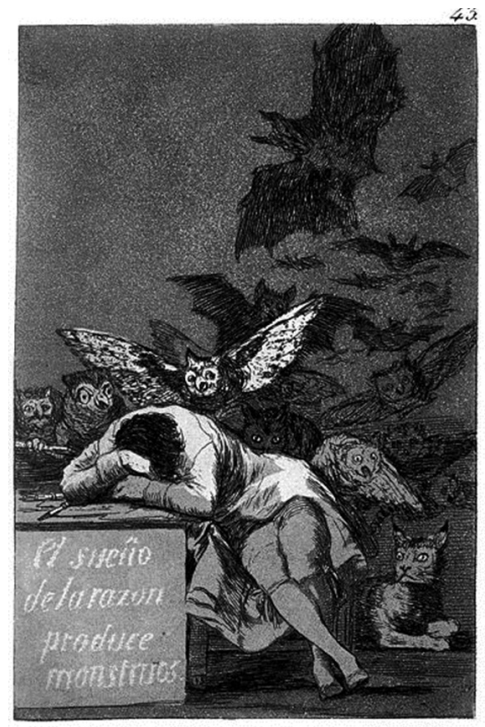

\section{Das Elementare und das Kollektiv}

Eines ästhetischen Prinzips wegen vollzieht der Schriftsteller und Literaturkritiker Einstein eine Wende zur Bildenden Kunst, die unmittelbar mit ihren Materialien arbeite - was er vergeblich auf das schriftstellerische Schaffen zu übertragen sucht. Erst die Surrealisten und mit ihnen Einstein stoßen - nicht zuletzt unter dem Einfluss Sigmund Freuds - bewusst zu der mythisch aktiven Schicht des Bewusstseins bzw. Unterbewusstseins vor, dem Traum. Hier unterliege, so meint man, die Zeichenproduktion noch nicht der o.g. Bewährungsprobe, sprich: der Zensur. Im Traum (auch in der écriture automatique) glaubt man Zugriff auf das Kollektiv zu erlangen, einem neuen Mythos zur Macht verhelfen zu können ${ }^{17}$ - ein unbedachtes

16. „El sueño de la razón produce monstruos“, span. „Der Schlaf/Traum der Vernunft gebiert Ungeheuer“.

17. In Folge der Verstrickung des Philologen in die ,Sauberkeit" des Zeichengebrauchs kann dieser nicht umhin - ,Wenn Moral; die des Handwerks.“ (BA 2, 469) -, auch mythische Reflexe im Kulturkampf der Gegenwart herauszustellen, vgl. die surrealistische These, der ein gewisser Tariq Ramadan an der Universität Straßburg zur Promotion verhalf, „le rêve est l'une des voies par lesquelles le divin 
messianisches Versprechen. Die Surrealisten glauben noch daran, ${ }^{18}$ als längst Andere die Macht tatsächlich ergriffen hatten und sich daranmachten, den Repräsentanten der „entarteten Kunst“ das Handwerk zu legen (Berufsverbot) und ihre Adepten ,jüdischer Rasse“ in Vernichtungslager einzuweisen. Die Handlanger des Regimes, die sich vor Gericht auf Befehlsnotstand beriefen, waren erbärmliche Opfer ihres eigenen Mythos, glaubten sie doch (auch) an ihre Mission.

Im mythischen Projekt der Moderne spielte die Kunst, insbesondere die Bildende Kunst, eine vorrangige Rolle, galt doch diese nicht mehr als ancilla ecclesiae, sondern das Kreative, inkarniert im proteischen Picasso (K 1, 69), erschien als zentrales Mythem - erschien, d.h. konnte in tausendfachen Werken auch unter Beweis gestellt werden. In der Postmoderne verflachte es freilich zu der Behauptung, dass jedermann/jedefrau Künstler sei. Der narrativen Willkür historisch gewachsener Religionen (Mythen) und ihrer Moralisierung begegnete der Surrealismus mit der Okkultation, sprich: Mythisierung, des Alltäglichen und des Zufalls, vor allem des Eros. Meine Absicht ist es nicht, dieses reizvolle Phänomen zu entzaubern, gleichwohl ist zu beobachten (angesichts der aktuellen Scheidungsraten), dass es zu einer Totalisierung wenig geeignet ist (oder dafür verantwortlich?). Überhaupt stellt sich die Frage, ob die longue durée, die (große) Mythen nun einmal zur Etablierung brauchen, ${ }^{19}$ in der „flüchtigen“ Moderne (Baudelaire) gegeben ist. Wir empfinden es heute kaum noch als Problem, dass unzählige Mythen (in den Medien) sprießen, ein neuer Mythos jedoch nicht in Sicht ist. Der Surrealismus war wohl der letzte (epigonale) Träger des fraglichen Postulats, mit dem Einstein schon 1931/32 Georges Braque ausklingen ließ - ich zitiere die veröffentlichte, französische Fassung:

L'accentuation romantique de l'irrationnel implique une primitivisation, ou, si l'on veut, une barbarisation. On ne se contente plus, enfin, de dérivations sublimes, de cette superstructure acquise à force de culture, et qui exclut les forces fondamentales de l'homme et du destin. Nous sommes de nouveau poussés par un besoin de destin et de contrainte. [...] Le mythe est réintégré dans la réalité, et le poème devient l'origine du Réel. $\left(\mathrm{GB}^{\mathrm{Zi}}, 140 \text {, dt. BA 3, 408f. }\right)^{20}$

Chiliastische Aussagen wie diese mögen Einstein veranlasst haben, seine Schrift, die erst 1934 erscheinen konnte, expressis verbis auf 1931-1932 zu datieren (GB ${ }^{\mathrm{Zi}}$,

inspire des messages ou des visions ou des réalités ou des vérités aux hommes“ (zit. L’OBS, Nr. 2766 vom 9.-15. November 2017, S. 39).

18. Vgl. Breton 1935: „[...] dans ces conditions, n'est-ce peut-être plus déjà de la création d'un mythe personnel qu'il s'agit en art, mais, avec le surréalisme, de la création d'un mythe collectif [...]“ (OC 2,439) sowie 1936/37: „Aucune tentative d'intimidation ne nous fera renoncer à la tâche que nous nous sommes assigné et qui est [...] l'élaboration du mythe collectif propre à notre époque [...].“ (OC 3, 667).

19. In der Regel wird die Verbreitung von großen Mythen (Religionen, Revolutionen) mit großem Blutvergießen Andersgläubiger begleitet; auch der Erste Weltkrieg - oder horribile dictu der Holocaust - wurde in diesem Sinne als „Reinigung“ verstanden.

20. „Le poème“ (dt. „Dichtung“) steht hier für die „Poetisation“ der Bildenden Kunst entsprechend der Arbitrarität des Sprachzeichens; s. Kiefer, Diskurswandel im Werk Carl Einsteins. Ein Beitrag zur Theorie und Geschichte der europäischen Avantgarde, Tübingen, Niemeyer (Communicatio. Studien zur europäischen Literatur- und Kulturgeschichte, Bd. 7), 1994, S. 449ff., korr. u. überarb. Fassung 2015 auf der home page der Carl-Einstein-Gesellschaft/Société-Carl-Einstein, [http://www. carleinstein.org] Stand 29. November 2017; hier auch weitere Literatur. 
10), nachdem ein anderes „Tausendjähriges Reich“ inzwischen seine Herrschaft angetreten hatte. Sei es, dass Braques Entwicklung für Einstein so vielversprechend war, sei es, dass Einsteins Ansichten für ihn selber einen so hohen Plausibilitätsgrad erreicht hatten, dass ein Umschlag vom subjektiven zum kollektiven Mythos bevorzustehen schien. Warum druckt Einstein dann aber die braquesche Graphik Odysseus (im Kontext der Theogonie-Serie) ${ }^{21}$ sowohl als Frontispiz seiner BraqueMonographie als auch an erster Stelle (außer der Reihe) der Abbildungen seines Katalogs zur großen Braque-Ausstellung, 9. April - 14. Mai 1933, in der Kunsthalle Basel (Abb. 2)? Mythische Figuration der List, kaum der Vernunft, repräsentiert Odysseus zwar keinen kollektiven Mythos, aber er scheint mir der ,Mythonaut', der den Weg ins ,romantische Intervall“ (GB ${ }^{\mathrm{Zi}}, 116$, das ,gelobte Land“) weisen sollte was dann freilich zu einer Irrfahrt wurde.

Abb. 2: Georges Braque: Odysseus, Pastel, 1932, Verbleib des Originals unbekannt, (c) Adagp

\section{Die Fabrikation des Mythos}

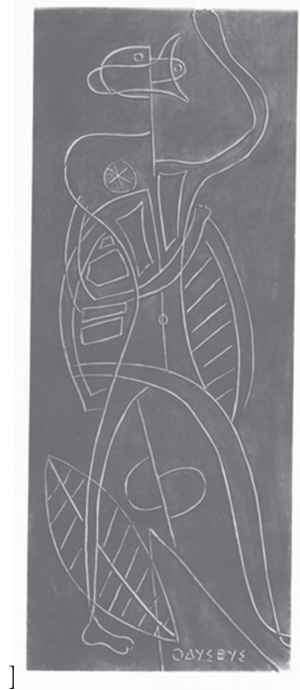

essant ist nun das Problem, dass Carl Einstein vermutlich zwischen 1934 und 1936 ,eine kritik und sociologie der moderne“ (Br. 394) entwirft, wie er im März 1936 an Vincenc Kramář schreibt, die allerdings nicht zum Druck gelangt, ${ }^{22}$ die aber die gesamte Avantgarde und auch seinen eigenen Beitrag aus den Angeln hebt, die schon genannte Fabrikation der Fiktionen. Einstein wollte

21. Die neueren Werkausgaben bzw. Einzelausgaben des Georges Braque haben das Frontispiz entweder abgedruckt und nicht kommentiert oder aber überhaupt völlig weggelassen. Einstein selber macht dazu zwei widersprüchliche Angaben, im Basler Katalog, Nr. 177 [S. 25]: Odysseus, Öl, 180 x 73 cm, 1932 bzw. in Georges Braque [S. 145]: Ulysse, Pastel, 1932.

22. Möglicherweise hat er noch in Spanien an einer Fassung gearbeitet. 
(musste!) sie wie zuvor schon Georges Braque ${ }^{23}$ ins Französische übersetzen lassen (als Fabrication des fictions), ging dann aber, bevor es dazu kam, nach Spanien.

Zwei Zwischenbemerkungen sind hier angebracht: (1) Georges Braque ist, wie Einstein selber gegenüber Ewald Wasmuth gesteht, „kein buch ueber braque“ (Br. 372); hatte er doch schon seit seinen Anfängen die Tendenz, seine Theorien an den Werken zu exemplifizieren, statt jene aus diesen zu entwickeln (freilich war immer eine intensive Phase des ,Sehens“ zwischen Bild und Wort geschaltet). Einstein, eng befreundet mit Braque, hatte diesen selber zu einer surrealistischen Schaffensphase angeregt, ${ }^{24}$ die aber keineswegs für dessen Gesamtwerk repräsentativ ist, als nämlich der Kunsthändler Ambroise Vollard dem Maler anbot, ein Buch nach seinem Gusto zu illustrieren. Braques Entscheidung für die Theogonie Hesiods wirkt für viele heute noch überraschend, weil man eben den Einfluss des Mythologen und Hesiod-Lesers Einstein nicht kannte (BA 3, 249f.). Somme toute kann daher Einsteins Georges Braque als surrealistische Ästhetik bezeichnet werden, wohl die ausgeprägteste der gesamten Bewegung. (2) In der Fabrikation der Fiktionen attackiert Einstein vor allem die Surrealisten als Vollender der europäischen Avantgarden (Vorstufen wie Dada übergeht er und - er schließt sich selber ein); er kontrastiert sie lediglich mit den Konstruktivisten, den „Salutisten der heilbringenden Quadrate“ (FF, 48). ${ }^{25}$

Dieser Widerruf besitzt - trotz der bei Einstein nicht ungewöhnlichen Polemik - einige Plausibilität, zumal er Erkenntnisse der vergangenen Jahre ${ }^{26}$ verarbeitet. Man kann den Text nicht als reines Pamphlet abtun. Das (deutsche) Typoskript wurde freilich erst 1973 herausgegeben und blieb weitgehend unbeachtet, auch weil das damit verknüpfte Editionsprojekt ${ }^{27}$ scheiterte. Namhafte Avantgarde- und insbesondere Surrealismus-Forscher in Deutschland und Frankreich kennen es ebenso wenig wie Georges Braque, so dass man sich nicht veranlasst sah, diesen fulminanten Umschlag von surrealistischer Ästhetik in Avantgarde- und Moderne-Kritik zu diskutieren. Den Zeitgenossen, die die Fabrikation nicht kennen konnten, ist kein Vorwurf zu machen, wenn auch ihre Schieflage - hinsichtlich des „mythe collectif“ (OC 2, 439) - nach 1933 peinlich zu erkennen ist. Der nationalphilologisch befangenen Forschung jedoch fehlt das dialektische Moment, das die Fabrikation der Fiktionen in eine Avantgarde-Debatte, eine Kritik der Moderne einbringt.

Die Fabrikation der Fiktionen folgt einer doppelten Dialektik. Einerseits nimmt Einstein Abschied von der mythologischen Verklärung Afrikas - „Ein Bordell von

23. Die zeitgenössische Übersetzung durch M. E. Zipruth $\left(\mathrm{GB}^{\mathrm{Zi}}\right)$ ist nicht hervorragend; das erhaltene deutsche Typoskript wurde von Jean-Loup Korzilius und Liliane Meffre neu ins Französische übersetzt $\left(\mathrm{GB}^{\mathrm{Ko}}\right)$.

24. Vgl. Maria Stavrinaki, „Les Braques de Carl Einstein. Entre stabilité classique et mythe romantique“, Georges Braque 1882-1963, Katalog Grand Palais, Paris, 16. September 2013 - 6. Januar 2014, S. 151-161.

25. Diese „fürchteten sich vor Traum und übersteigerter Sexualität, die [im Surrealismus] en vogue waren" (FF, 48) Schon in seinem autobiographischen Gedicht, Entwurf einer Landschaft nimmt Einstein 1930 am Konstruktivismus Anstoß (BA 3, 73, V. 1-6).

26. Vor allem semiotische und stilistische, aber auch psychoanalytische und soziologische Erkenntnisse.

27. Der Text wurde nicht in die Medusa- bzw. Berliner Ausgabe übernommen und bislang auch nicht ins Französische übersetzt. 
Spirits lärmte, wenn Bumbo sich vergnügte.“ (FF, 167) -, andererseits exemplifiziert er seine „Ethnologie du Blanc“, ${ }^{28}$ die er seinen fremdkulturellen Studien abgewonnen hatte, an der modernen Kunst und ihrer Rezeption - eine Inversion in der Art von The Savage Hits Back. ${ }^{29}$ Künstler und Intellektuelle agieren als „Fetischeure des Geistes“ (FF, 145), ihre Klientel lässt sich mit infantilen und sexuellen Traumphantasien betäuben und von der politischen Realität ablenken. Energisch bestreitet Einstein nunmehr, dass sich auf dieser Basis ein kollektiver Mythos bilden ließe. Jede Übereinkunft (Konvention) fehle.

Eine Surrealismus-Debatte nach Art der deutschen Expressionismus-Debatte (die freilich auch schon geschichtlich ist und keineswegs musterhaft) erfolgte in Frankreich erst ums Jahr 2003, als Jean Clair seinen Essay ${ }^{30}$ in die Diskussion einbrachte - und Jean Clair hatte Einsteins Fabrikation der Fiktionen gelesen! ${ }^{31}$ Längst hatte sich aber der Surrealismus in die Geschichtsbücher eingeschrieben und die Klassische Moderne war von der Kulturindustrie vereinnahmt. Kann und soll an diesen Bildungsgütern gerüttelt werden?

Wenn Einstein Mitte der 30er Jahre mit einem Schlag widerruft, was er von den ersten Kapiteln des Bebuquin (1906) bis zur dritten Auflage der Kunst des 20. Jahrhunderts (1931) aufgebaut hatte, dann kann man wohl fragen, ob etwas seinem Autodafé widerstanden hat. Eine Spekulation gewiss, aber Fernand Léger ist der einzige Künstler, den Einstein - „mit Mühe kaum“ - dem ursprünglich primitivistischen Kubismus (BA 1, 240 u. 245) zurechnet, ${ }^{32}$ dessen Werk aber eine hochgradige Kollektivität aufweist. Kein Wunder, dass sich die einzige öffentliche Attacke des Breton-Kreises gegen Einstein (aus der Feder René Crevels) gerade an dessen Ausführungen zu Léger, insbesondere zum légerschen Tektonismus, entzündet. ${ }^{33}$ Die tektonische Zensur, die Einstein für die Gestaltung des Kunstwerks verantwortlich macht, ist ja in der Tat ein wichtiges Unterscheidungsmerkmal zwischen dem einsteinschen und dem bretonschen Surrealismus.

Man darf auch annehmen, dass die Fabrikation der Fiktionen nicht das letzte Wort Einsteins zur Avantgarde gewesen wäre, zumal die Fassung letzter Hand nicht zu bestimmen ist. Seine Entwürfe zu einer Geschichte der Kunst, insbesondere der

28. So in einem Interview mit B. J. Kospoth (,A New Philosophy of Art“, Chicago Sunday Tribune. European Edition, Nr. 4932 vom 18. Januar 1931, S. 5).

29. Julius E. Lips, The Savage Hits Back or The White Man Through Natives Eyes, Einf. von Bronislaw Malinowski, Übers. von Vincent Benso, London, Lovat Dickson, 1937.

30. Jean Clair, Du surréalisme considéré dans ses rapports au totalitarisme et aux tables tournantes. Contribution à une histoire de l'insensé, Paris, Arthème Fayard, 2003.

31. Zumindest insofern Liliane Meffre in ihrer Dissertation (Carl Einstein et la problématique des avantgardes dans les arts plastiques, Bern u.a., Peter Lang, 1989 [Contacts III, Bd. 8]) den Text zitierte und ins Französische übersetzte, Clair kennt noch nicht Meffres umfassendere Studie Carl Einstein 18851940. Itinéraires d'une pensée moderne, Paris, Presses de l’Université de Paris-Sorbonne, 2002.

32. Einstein plante mit Léger eine - futuristische - „Automatengeschichte“(Br. 99), bei seiner Abneigung gegenüber dem Futurismus (als Modernismus) verwunderlich; vgl. Kiefer, „Primitivismus und Modernismus im Werk Carl Einsteins und in den europäischen Avantgarden“, in Nicola Creighton u. Andreas Kramer (Hrsg.), Carl Einstein und die europäische Avantgarde / Carl Einstein and the European Avant-Garde, Berlin/Boston, de Gruyter (spectrum Literaturwissenschaft / spectrum Literature, Bd. 30), 2012, S. 186-209, hier S. 207.

33. René Crevel, „Critique d'Art“, Le Surréalisme au service de la Révolution, Nr. 1 (1930), S. 12. 
modernen Kunst, habe ich schon erwähnt; er will auch zur ,esthétique expérimentale“ weiterarbeiten, wie er an die American Guild for German Cultural Freedom 1940 schreibt (Br. 420). Er will vor allem schreiben, nach dem Sieg über den Faschismus in Spanien: „faire des bouquins solides, loin de tous les penchants des modernes et des bien pensants de tous les avant-gardes, des livres durs et comiques" (Br. 416). Zum Vergleich denkt er an Gulliver's Travels, Don Quichotte, Bouvard et Pécuchet, und er fügt den illustren Namen - schon in einem Brief an Tony Simon-Wolfskehl von 1923 - hinzu: „Vielleicht Bebuquin - wenn er fertig ist. Sonst hat es sich auch nicht gelohnt. Vielleicht bin ich grössenwahnsinnig aber ich spreche nur von Absicht nicht Gelingen. Der Teufel hole das Metier.“ (Br. 175)

Während die Bewegung des Surrealismus konsequent ihrer Historizität zurollt, lockern sich dessen Selbstdefinitionen, werden auch die Interpreten weniger befangen. Überblickt man nun die surrealistische Ästhetik Einsteins (in Georges Braque) und seine Surrealismus-Avantgarde-Moderne-Kritik in der Fabrikation der Fiktionen, so drängt sich das Desiderat eines übergreifenden Konzepts auf, um die banale Frage „Wer hat denn nun recht?“ zu vermeiden. Gehen wir den Wortspielen aus dem Weg, dass der Surrealismus selber ein Mythos sei oder der gesuchte Mythos der fromme Wunsch eines verkappten Religionsstifters, eines „Cagliostro der Modernität" (KSA 6, 357). Zuzustimmen ist Jacqueline Chénieux-Gendron und Yves Vadé jedenfalls, die eine Abteilung ihres einschlägigen Kolloquienbandes mit „Le mythe introuvable“34 überschreiben. War also alles umsonst? Trotz alledem hat sich das mythische Projekt ja durchgesetzt: zwar um den Preis seiner Kommerzialisierung und Musealisierung (und einigem Gelehrtenschweiß), die den Sieg seiner schlimmsten Feinde, des Bürgertums und des Kapitalismus, voraussetzen. So gesehen beschreibt Einsteins Fabrikation der Fiktionen eigentlich die paradoxe Erfolgsgeschichte des Surrealismus. Aber mit Goethe zu sprechen: Wir wollten in der Tat „die Umwälzungen nicht wünschen“, ${ }^{35}$ die in Europa einen kollektiven Mythos in Kraft gesetzt hätten. Der dekorativen Qualität der Werke wegen bleiben die im Kern explosiven weltanschaulichen Konflikte jedoch verborgen.

\section{Mythos und Exil}

Als sich Einstein von der Weltwirtschaftskrise und dem Nationalsozialismus ruiniert im Exil wiederfindet - war er doch 1928 nur von Berlin nach Paris umgezogen ${ }^{36}$ -, verklärt er diesen Zustand nicht mythisch (hebr. Galut), obwohl er seine „Heimatlosigkeit“ als eine spezifisch jüdische reflektiert (reflektieren muss) -

34. Jacqueline Chénieux-Gendron u. Yves Vadé (Hrsg.), Pensée mythique et surréalisme, Paris, Lachenal \& Ritter (Pleine Marge, Nr. 7), 1996, S. $23 \mathrm{ff}$.

35. Johann Wolfgang Goethe, „Literarischer Sanscülottismus“: „Wir wollen die Umwälzungen nicht wünschen, die in Deutschland klassische Werke vorbereiten könnten." (MA 4.2, 17)

36. Daher übersieht ihn die Exilforschung gerne. Einstein war jedoch die ganzen 20-er Jahre über in Berlin antisemitischen Anfeindungen ausgesetzt 
allerdings ohne religiöse Konnotationen. ${ }^{37}$ Am 18. Februar 1933 notiert er: ,ich sehe, immer mehrwerde ich allein sein, jude, deutschsprechend, in frankreich. jude ohne gott und ohne kenntnis unserer vergangenen zeit [...].“(CEA, 81) Daher findet er keinen Halt in einem kollektiven Mythos, etwa dem des Judentums; doch der Sachverhalt ist komplex: Einstein zieht zwar kurzentschlossen in den antifaschistischen Kampf nach Spanien: „dans les temps qui courent le fusil est nécessaire pour compenser la lâcheté du stylo“ (Br. 416), doch in der Kolonne Buonaventura Durrutis erlebt er wiederum ein - nunmehr syndikalistisch-anarchistisches - Gemeinschaftsideal, eine „verschworene“ mythische Gemeinschaft. In seiner Rundfunkansprache zum Tod des charismatischen Führers versucht er, der Problematik halb bewusst, Durrutis personalen Mythos nicht weiter zu verbreiten:

Durruti, dieser außergewöhnlich sachliche Mann, sprach nie von sich, von seiner Person. Er hatte das vorgeschichtliche Wort ,ich“ aus der Grammatik verbannt. In der Kolonne Durruti kennt man nur die kollektive Syntax. Die Kameraden werden die Literaten lehren, die Grammatik im kollektiven Sinn zu erneuern. (BA 3, 520) ${ }^{38}$

Dass er dieses Vorhaben nach seiner Rückkehr aus Spanien noch verfolgt hat, ist angesichts seiner Notlage und dann seiner Deportation in ein französisches Lager (Bassens) wenig wahrscheinlich. Ohne Zweifel hatte er aber in zahllosen Notizen bis zu seinem Aufbruch nach Spanien Material zu einer Fortsetzung des Bebuquin (BEB II) gesammelt. Dieses Material ist zwar noch stärker autobiographisch eingefärbt als der abstrakte Romanerstling, ${ }^{39}$ „Bebuquin“ gehört jedoch eindeutig in die Kategorie des personalen Mythos. Was sein Autor längst schon fühlt - „BEB EIN DON QUICHOTE“ (CEA, 51) -, überträgt er in der Fabrikation der Fiktionen auf die gesamte Avantgarde:

Die Geschichte der Intellektuellen ist die Chronik einer lächerlichen Donquichoterie, eines archaischen Wahns [Mythos]. Die Literaten behaupteten immer noch, die Vision gebäre die Fakten. Aller Modernität zum Trotz blieben sie in überalterte Magie gebannt. (FF, 233)

\section{Fazit}

Die Aufklärung hatte ihr Werk der Entmythologisierung keineswegs so weit getrieben, dass es nicht eines Nietzsche bedurft hätte und jener „Urkatastrophe“ des 20. Jahrhunderts, um Europa seiner metaphysischen Sekurität zu berauben und in

37. Vgl. Kiefer, „Carl Einstein“, in Andreas B. Kilcher (Hrsg.), Metzler Lexikon der deutsch-jüdischen Literatur. Jüdische Autorinnen und Autoren deutscher Sprache von der Aufklärung bis zur Gegenwart, Stuttgart, Metzler, 2012 (2., akt. u. erw. Aufl.), S. 129-131.

38. Die Ansprache wurde vermutlich in französischer Sprache gehalten, doch in der Veröffentlichung fehlt genau dieser Passus, vgl. Kiefer, ,,Lingua - Signe, mythe, grammaire et style dans l'œuvre de Carl Einstein“, [http://melusine-surrealisme.fr/wp/?p=2287], Stand 29. November 2017.

39. Vgl. Kiefer: „Bebuquins Kindheit und Jugend - Carl Einsteins regressive Utopie“, in Historiographie der Moderne - Carl Einstein, Paul Klee, Robert Walser und die wechselseitige Erhellung der Künste, hrsg. von Michael Baumgartner, Andreas Michel, Reto Sorg, Paderborn, Fink, 2016, S. 105-120. 
eine Krise „transzendentaler Obdachlosigkeit“" ${ }^{* 40}$ zu stürzen. Zugleich hatte derselbe Prozess der Modernisierung, der die Waffen des Ersten Weltkriegs schmiedete, Kräfte des Individuums freigesetzt, die sich schöpferisch - wenn auch größtenteils unverstanden - in den Künsten der Avantgarde entfalteten. Aber just von dieser Seite her sollte eine gesellschaftliche Erneuerung, elementar und primitiv, ansetzen, ein neuer - namenloser - Mythos entstehen. Und es sollte nicht nur ein „,personaler Mythos“ sein, denn Mythos impliziert notwendigerweise Kollektivität. Diese freilich musste mit mehr oder weniger messianischen Anteilen „organisiert“ werden, konnte nicht über Jahrtausende wachsen. Und was wollte man auch mit den Mythen Afrikas oder Ozeaniens, deren primitive Formen zwar inspirierten, aber, inhaltlich gesehen, unzeitgemäßen Aberglauben boten? Bis 1933 vermochte Einstein freilich nicht die Verwissenschaftlichung des Mythos-Begriffs, die er wie etwa Ernst Cassirer ${ }^{41}$ energisch betrieb, aus seinem utopischen Denken zu entflechten.

Einer der fatalsten Begriffe des 20. Jahrhunderts ist der der Gemeinschaft. Er wird von allen politischen Richtungen, links wie rechts, in Anspruch genommen (und hielt nie seine Versprechen). Führer beanspruchten Deutungshoheit, auch im ästhetischen Lager: Marinetti, Breton - nicht zu vergessen, wiederum alles negierend, Dada!

In diesem Rahmen agiert Carl Einstein: Je nach Weltlage setzt er, sei es auf die ästhetische Primitive, den Kubismus, dann den Surrealismus, sei es auf die politische Primitive, den Kommunismus. Fusionen misslingen. Als der stärkere Mythos, dem sich die frustrierten Massen willig unterwerfen, in Deutschland obsiegt, der Nationalsozialismus, kämpft Einstein dagegen an. Im „kurzen Sommer der Anarchie“ (Hans Magnus Enzensberger) flackert sein Utopismus ein letztes Mal wieder auf, obwohl er längst erkannt hat, dass weder aus der ästhetischen Gestaltung der Krise, noch aus der politischer Partizipation der erwünschte soziale Zusammenhalt entsteht. Im politischen Exil begreift Einstein, dass er, der Intellektuelle und Romanschreiber, nie „dazu“ gehört hat, dass er immer Emigrant gewesen ist. Für seine Reflexionsfigur Bebuquin notiert er am 31. Januar 1934:

BEB versteht nun, daß er sein ganzes leben nur einer abgetrennten clique angehört hat, die in ästhetischer eitelkeit und erfolgssucht untereinander sich bekämpfte; also BEB war, wie er jetzt sieht, sein ganzes leben emigrant gewesen. Sein ganzer effort war daraufhin gegangen emigrant, d.h. isoliert und ausnahme zu sein. immer war er auf einer flucht vor einem bindenden milieu gewesen. (CEA, 52)

\section{Abgekürzt zitierte Literatur}

BA 1, 2, 3 = Carl Einstein, Werke. Berliner Ausgabe, 3 Bde., hrsg. von Hermann Haarmann u. Klaus Siebenhaar, Berlin, Fannei \& Walz, 1994 u. 1996.

40. Georg Lukács, Die Theorie des Romans. Ein geschichtsphilosophischer Versuch über die Formen der großen Epik, Darmstadt/Neuwied, Luchterhand, 1971 (1916), S. 32.

41. Ernst Cassirer, Philosophie der symbolischen Formen, 2. Teil: Das mythische Denken, Darmstadt, Wissenschaftliche Buchgesellschaft, 1964 (1924). 
$\mathrm{Br}=$ Carl Einstein Briefwechsel 1904-1940, hrsg. von Klaus H. Kiefer u. Liliane Meffre, Stuttgart, Metzler, ca. 2020 (Die Zahl hinter dem Komma bezeichnet die Nr.).

CEA = Carl-Einstein-Archiv, Akademie der Künste, Berlin (digitalisiert: [https:// archiv.adk.de]).

EA = Europa-Almanach. Malerei, Literatur, Musik, Architektur, Plastik, Bühne, Film, Mode, außerdem nicht unwichtige Nebenbemerkungen, hrsg. von Carl Einstein u. Paul Westheim, Potsdam, Kiepenheuer, 1925.

$\mathrm{FF}=$ Carl Einstein, Die Fabrikation der Fiktionen. Gesammelte Werke in Einzelausgaben, Bd. 4 (mehr nicht ersch.), eingel. von Helmut Heißenbüttel, hrsg. von Sibylle Penkert, m. Beitr. von ders. u. Katrin Sello, Reinbek bei Hamburg, Rowohlt, 1973.

$\mathrm{GB}^{\mathrm{Zi}}=$ Carl Einstein, Georges Braque, ins Frz. übers. von M. E. Zipruth, Paris,

Chroniques du jour/London, Anton Zwemmer/New York, Erhard Weyhe, 1934 $\left(\mathrm{XX}^{\mathrm{e}}\right.$ siècle, $\left.\mathrm{Bd} .7\right)$.

$\mathrm{GB}^{\mathrm{Ko}}=$ Carl Einstein, Georges Braque, hrsg. von Liliane Meffre, ins Frz. übers. von Jean-Loup Korzilius, Bruxelles, La Part de l'Eil, 2003.

K 1, 2, 3 = Carl Einstein, Die Kunst des 20. Jahrhunderts. Propyläen Kunstgeschichte,

Bd. 16, Berlin, Propyläen Verlag, 1926, 1928, 1931 (2. u. 3., jeweils veränd. u. erw. Aufl.).

$\mathrm{KSA}=$ Friedrich Nietzsche, Sämtliche Werke. Kritische Studienausgabe in 15 Bdn., hrsg. von Giorgio Colli u. Mazzino Montinari, München/Berlin/New York, de Gruyter u. dtv, 1980.

MA = Johann Wolfgang Goethe, Sämtliche Werke nach Epochen seines Schaffens.

Münchner Ausgabe, hrsg. von Karl Richter u.a., Bd. 4.2: Wirkungen der Französischen Revolution 1791-1797, hrsg. von Klaus H. Kiefer u.a., München, Hanser, 1986.

OC $=$ André Breton, Euvres complètes, 4 Bde., hrsg. von Marguerite Bonnet u.a., Paris, Gallimard (Bibliothèque de la Pléiade), 1988-2008.

W 4 = Carl Einstein, Werke, Bd. 4: Texte aus dem Nachlaß I, hrsg. von Hermann Haarmann u. Klaus Siebenhaar, Berlin/Wien, Fannei \& Walz, 1992.

\section{Abbildungsverzeichnis}

Abb. 1: Francisco de Goya: Capricho, Nr. 43: El sueño de la razón produce monstruos, Aquatinta-Radierung 21,3 x 15,1 cm, 1799, Museo del Prado, Madrid

Abb. 2: Georges Braque: Odysseus, Pastel, 1932, Verbleib des Originals unbekannt, (C) Adagp 
